\title{
Tsafon
}

Revue d'études juives du Nord

$74 \mid 2017$

La Déclaration Balfour, vers l'État d'Israël ?

\section{Portraits des protagonistes de la Déclaration Balfour}

\section{Philippe Boukara}

\section{(2) OpenEdition \\ 1 Journals}

Édition électronique

URL : https://journals.openedition.org/tsafon/396

DOI : $10.4000 /$ tsafon.396

ISSN : 2609-6420

Éditeur

Association Jean-Marie Delmaire

\section{Édition imprimée}

Date de publication : 1 décembre 2017

Pagination : $39-48$

ISSN : 1149-6630

\section{Référence électronique}

Philippe Boukara, « Portraits des protagonistes de la Déclaration Balfour », Tsafon [En ligne], 74 | 2017, mis en ligne le 31 mai 2018, consulté le 21 septembre 2021. URL : http://journals.openedition.org/ tsafon/396 ; DOl : https://doi.org/10.4000/tsafon.396 


\section{Portraits des protagonistes de la Déclaration Balfour}

Philippe Boukara*

\section{L'auteur de la Déclaration : Arthur Balfour (1848-1930)}

Lord Arthur Balfour est né en 1848 dans le village écossais de Whittingehame. Fils et petit-fils de député, député lui-même à partir de 1874, il est apparenté à des hommes politiques importants dans les deux branches de sa famille - son oncle, Lord Salisbury (1830-1903), a été Premier ministre à trois reprises. Formé à la philosophie, célibataire toute sa vie, frère d'un grand biologiste ${ }^{1}$ considéré comme le successeur de Darwin, il est bien représentatif d'un état d'esprit non-conformiste très influent dans l'élite du Royaume-Uni et qui n'est peut-être pas sans rapport avec la publication de la fameuse Déclaration.

La fonction la plus importante qu'il ait exercée est celle de Premier ministre, entre 1902 et 1905 , au titre du parti tory. Pendant cette période, Balfour conclut l'Entente cordiale avec la France (1904), mais il fait aussi voter, le 2 mai 1905, l'Aliens Act loi instituant le contrôle et l'enregistrement administratifs de l'immigration, dont le but avoué était de restreindre l'arrivée des Juifs de l'Empire russe venus nombreux à partir des pogromes des années 1880. La responsabilité prise par Balfour à cette occasion a donné lieu à de nombreux commentaires concernant la nature de ses sentiments envers les Juifs. C'est peu après ce vote qu'il rencontre le jeune Chaïm Weizmann, dans le contexte de la campagne électorale où il est battu dans sa circonscription de Manchester.

\footnotetext{
* Mémorial de la Shoah et Collège des Bernardins.

${ }^{1}$ Francis Balfour (1851-1882), mort dans un accident d'alpinisme sur le Mont-Blanc.
} 
Chef de l'opposition jusqu'en 1911, Balfour revient au gouvernement en mai 1915 comme Premier Lord de l'Amirauté dans le cabinet de coalition dirigé par le libéral H. H. Asquith (1852-1928), puis en décembre 1916, comme secrétaire au Foreign Office dans le nouveau cabinet de coalition dirigé par Lloyd George (1863-1945), libéral lui aussi. À ce dernier poste, il est amené à séjourner aux États-Unis en avril 1917 pour consolider l'alliance avec ce pays. Il est aussi mêlé, bien sûr, aux négociations du traité de Versailles, puisque c'est en octobre 1919 qu'il change d'attributions pour devenir Lord Président du Conseil. Il détient ce portefeuille d'influence jusqu'en 1922, toujours en coalition avec les libéraux, puis à nouveau de 1925 à 1929, cette fois dans le gouvernement conservateur de Stanley Baldwin (1867-1947).

À l'occasion de l'inauguration de l'Université hébraïque de Jérusalem, en avril 1925, Arthur Balfour effectue une visite en Palestine qui lui permet de constater l'étendue de sa popularité dans le monde juif. Le mochav (village coopératif) de Balfouria a été fondé en son honneur en 1922, dans la vallée du Jezreel. Le premier mochav, Nahalal, a été fondé en 1921, et Balfouria est le troisième village créé sur ce modèle situé près d'Afoula en Galilée, le mochav existe toujours, abritant notamment une réserve naturelle d'un demi-hectare, et comptait 445 habitants en 2016.

Lord Balfour, joueur de tennis confirmé, a connu une longévité ministérielle et parlementaire exceptionnelle. Il est enterré en 1930, selon son vœu, dans son village natal. Entre 1939 et 1941, à l'initiative de son neveu Robert Arthur Lytton (1902-1968), la maison de son enfance a servi de ferme-école sioniste pour l'accueil de 160 enfants et adolescents juifs réfugiés, arrivés d'Europe centrale dans le cadre de ce que les Britanniques appellent les Kindertransports. C'est surtout sa nièce Blanche Dugdale (1880-1948) qui s'est fortement engagée, par la parole et par l'écrit, en faveur du sionisme, au point de travailler à plein temps à Londres pour le département politique de l'Agence juive à partir de 1940 . Surnommée Baffy, cette ancienne fonctionnaire du Renseignement naval a coopéré étroitement avec Chaïm Weizmann en l'aidant de ses relations dans les milieux de la Société des Nations. Blanche Dugdale est décédée le 15 mai 1948, après avoir appris la création, le 14 mai, de l'État d'Israël - son journal personnel a été déposé dans les archives Weizmann. Précisons que son mari, Edgar Dugdale (1876-1964), est l'auteur de la première traduction en anglais de Mein Kampf, effectuée dans un souci militant antinazi. Précisons encore que de nombreux garçons juifs dans le 
monde nés en 1930 ont reçu pour prénom Arthur - un geste de mémoire qui ne trompe pas...

\section{Le destinataire de la Déclaration : Lord Lionel Walter Rothschild (1868-1937)}

Destinataire de la Déclaration Balfour le 2 novembre 1917, Lord Lionel Walter Rothschild (1868-1937) a légitimement sa place dans l'historiographie du sionisme. C'est en raison du prestige de son nom et de sa famille que Chaïm Weizmann a souhaité l'associer à ses démarches préliminaires à la Déclaration Balfour - il a fait en sorte, d'ailleurs, qu'elle soit envoyée à son adresse du 148 Piccadilly Street. Le baron est en effet le petit-fils de Lionel Nathan Rothschild, premier Juif siégeant comme député à la Chambre des Communes en 1858. Son père, le baron Nathaniel Rothschild (1840-1915), député aux Communes à partir de 1865 et premier Juif non converti à entrer à la Chambre des Lords, était considéré comme le principal dirigeant du judaïsme britannique - il n'était pas favorable au sionisme même s'il avait été impressionné par la personnalité de Theodore Herzl.

Lord Lionel Walter, lui-même député tory de 1899 à 1910, accepte de participer, le 17 février 1917, à une importante réunion au domicile du grand rabbin Moses Gaster au cours de laquelle Chaïm Weizmann et ses principaux soutiens élaborent une stratégie pour négocier avec le gouvernement britannique ${ }^{2}$. James de Rothschild, le fils du baron parisien Edmond, est là (lui et son père sont très fortement engagés dans les activités pro-palestiniennes, selon les termes de l'époque). Présents aussi l'homme d'État libéral Herbert Samuel, le dirigeant sioniste russe Nahum Sokolov, ses homologues britanniques Joseph Cowen et Herbert Bentwich, et également, à titre privé, le diplomate Mark Sykes - dont le nom est attaché aux accords Sykes-Picot. Lord Rothschild envoie peu après une lettre au Times pour répondre aux arguments des grands notables juifs de tendance assimilationniste comme Edwin Montagu, nouveau Secrétaire aux affaires de l'Inde. En juin, avec Sir Ronald Graham, expert du Foreign Office, il accompagne Weizmann chez Balfour pour lui demander explicitement de faire une déclaration - ce dernier en accepte le principe et demande qu'on lui soumette un projet. C'est Lord Rothschild encore qui transmet le 18 juillet le projet élaboré par les sionistes. En septembre, il indique à Weizmann qu'il a écrit à

\footnotetext{
${ }^{2}$ Chaïm Weizmann, Naissance d'Israël (Trial and Error), Paris, Gallimard, 1957, sq.
} 
Balfour pour lui demander audience : les deux hommes se voient le 21 septembre, et il en rend compte immédiatement à Weizmann. C'est lui, enfin, qui préside en juillet 1920 une imposante assemblée d'hommage à Balfour au Albert Hall de Londres, en marge de la première Conférence sioniste internationale de l'après-guerre.

À vrai dire, si Lord Lionel Walter est resté célèbre, c'est pour une tout autre raison que son engagement, bref somme toute, en faveur du sionisme. C'est en tant que zoologiste passionné qu'il s'est distingué par ses collections d'oiseaux et de papillons du monde entier, d'un volume et d'une qualité exceptionnels : 300000 oiseaux, 200000 œufs d'oiseaux, 2250000 papillons, 30000 coléoptères, ainsi que des milliers de spécimens de mammifères, de reptiles et de poissons. Il créa un musée de zoologie à Tring, au nord de Londres, qui est aujourd'hui intégré au Musée d'histoire naturelle de la capitale britannique : sa donation est la plus importante que le British Museum ait jamais reçue. Un étourneau et une girafe ont été baptisés de son nom, ainsi que 153 insectes, 58 oiseaux, 17 mammifères, trois poissons, trois araignées, deux reptiles, un mille-pattes et un ver. Réputé excentrique - ce qui est plutôt une qualité dans la culture anglaise - Lord Rothschild a voulu montrer que les zèbres pouvaient être apprivoisés en conduisant à Buckingham Palace un charriot attelé à ces animaux.

Pour en revenir à la Déclaration Balfour, on donnera ici, à titre documentaire, une traduction de la lettre envoyée par Lord Rothschild le 4 novembre 1917 , après réception de ce document ${ }^{3}$.

Cher M. Balfour,

J'écris pour vous remercier très sincèrement pour votre lettre et aussi pour le grand intérêt que vous avez montré pour les vœux de la grande masse du peuple juif et aussi pour vos efforts et la peine que vous vous êtes donné en leur faveur. Je peux vous assurer que la gratitude de dix millions de personnes vous sera acquise, car le gouvernement britannique a ouvert, par son message, une perspective de sécurité et de confort à une grande masse de gens qui en ont besoin. Je me permets de croire que vous en avez été informé - déjà, dans de nombreuses régions de Russie, une persécution renouvelée a éclaté. Avec mes remerciements renouvelés pour vous et pour le Gouvernement de Sa Majesté, je reste

Sincèrement votre

Rothschild.

${ }^{3}$ Fac simile dans L.J.S., "Balfour Declaration», Cecil Roth (dir.), Encyclopaedia Judaica, Keter, Jerusalem, 1972, vol. XVI, pp. 135-136. 


\section{Le négociateur de la Déclaration : Chaïm Weizmann (1874-1952)}

Évoquer Chaïm Weizmann, principal négociateur sioniste de la Déclaration Balfour, c'est évoquer le personnage qui incarne l'une des trois étapes fondamentales de l'histoire du sionisme: après Theodore Herzl, qui créa la structure internationale du mouvement sioniste revendiquant un « foyer » en Palestine pour le peuple juif, avant David Ben Gourion, qui proclama l'indépendance de l'État d'Israël et eut la charge de consolider son existence pendant ses quinze premières années, c'est Weizmann qui eut à parcourir la longue distance allant de la théorie abstraite à sa réalisation concrète. C'est justement la Déclaration Balfour qui marqua le choix historique d'une des grandes puissances mondiales de s'engager (très prudemment, on le sait) en faveur dudit «foyer », et surtout celui du mouvement sioniste de parier sur un partenariat avec le Royaume-Uni pour parvenir à ses fins. Le regard distancié de l'historien doit permettre d'évaluer avec toutes les nuances nécessaires les avantages réels que le mouvement sioniste a tirés de ce partenariat jusqu'à la fin des années 1930, et le préjudice tout aussi réel subi après 1939 du fait de la puissance mandataire en Palestine. Weizmann a voué sa vie à cette option anglo-sioniste qui a rapproché le sionisme de son but final, il n'était pas l'homme à couper dans la violence finale les liens qu'il avait lui-même noués.

Né dans le shtetl de Motol en Biélorussie, il est un exemple idéal et typique de ce que la Haskala, le mouvement juif des Lumières, a produit dans sa version est-orientale : un intellectuel profondément imprégné de tout le patrimoine spirituel juif et en même temps ouvert aux formes les plus avancées de la culture occidentale moderne. Contraint comme tant d'autres par le numerus clausus de quitter son pays natal pour faire ses études supérieures, il est l'un des animateurs de la puissante Association des étudiants juifs russes à l'université de Berlin dans les années 1890, dans laquelle se retrouvent de grands esprits qui jouèrent un rôle dans l'histoire du sionisme. Il est de la génération des Hovevei Zion, les Amants de Sion, qui ont précédé la formation de l'Organisation sioniste par Theodore Herzl. C'est pourquoi il est, à partir de 1901, le principal animateur de la fraction démocratique, opposition interne à Herzl qui regroupe de brillants intellectuels comme Martin Buber. Ces jeunes hommes sont en réalité des disciples de Ahad Ha'am (1856-1927), le penseur du «sionisme culturel», mentor des Hovevei Zion d'Odessa, 
installé à Londres à partir de 1908. Du fait de sa spécialisation comme chimiste (il est passé par les universités suisses à partir de 1897, puis a été nommé professeur à Manchester en 1904), Weizmann inventa le concept de « sionisme synthétique » pour concilier la démarche politicodiplomatique de Herzl et celle d'Ahad Ha'am, centrée sur la vie de l'esprit.

Dans le sillage d'Ahad Ha'am, Weizmann a toujours mis en avant la culture, la science et la qualité de l'éducation comme le fondement de la pérennité du futur État juif. C'est pourquoi on doit le créditer des efforts continus qui ont permis de créer l'Université hébraïque de Jérusalem (1925). Mais on lui doit aussi l'Institut Weizmann, créé à partir de 1934 dans sa propre résidence de Rehovot, au sud de Tel-Aviv. Qui pourrait nier que l'État d'Israël d'aujourd'hui, malgré beaucoup d'incertitudes notamment géopolitiques, tire une bonne part de sa force de ses universités et de ses centres de recherche? Fréquentant quotidiennement les universitaires, Weizmann groupa autour de lui, à Londres où il vint résider pendant la Première Guerre mondiale, une équipe de grands professeurs juifs idéalistes qui donnèrent un éclat particulier au travail politique qu'ils menèrent à bien : Selig Brodetzky (1888-1954), mathématicien, Lewis Namier (1888-1960), historien, David Eder (1865-1936), psychanalyste, notamment, furent ses proches collaborateurs. Weizmann a pu, grâce aux brevets déposés au fur et à mesure de son travail de chercheur, acquérir une complète indépendance financière, lui évitant d'être un politicien professionnel, et a eu l'habitude de fréquenter des industriels et des financiers dont il savait gagner la confiance. Ses relations avec les dirigeants de Marks and Spencer, par exemple - Harry Sacher et son beau-frère Sir Simon Marks, établis à Manchester - furent de complicité véritable.

Weizmann est élu président de la Fédération sioniste de GrandeBretagne quelques jours seulement avant la Déclaration Balfour. C'est en juillet 1920 qu'il est investi à la présidence de l'Exécutif sioniste sur le plan mondial - fonction qu'il exerçait déjà de fait depuis ladite Déclaration, les sionistes du camp germano-autrichien ayant été pris de vitesse. Jusqu'en 1931, il applique ses méthodes graduelles et pragmatiques qui lui valent bien des critiques, créant en 1920 le Keren Hayessod, organe de collecte de fonds, et en 1929, l'Agence juive pour la Palestine, pour élargir les soutiens au-delà du mouvement sioniste organisé. Au Congrès sioniste de 1931, il est mis en minorité par Jabotinsky et Ben Gourion coalisés, parce que les émeutes arabes de 
1929 ont failli provoquer l'annulation de la Déclaration Balfour et qu'il a dû sauver la situation au prix de beaucoup de diplomatie. Il est cependant très actif en 1933 pour faire face à l'urgence des premières vagues d'émigration des Juifs d'Allemagne. Son retour à la présidence en 1935, avec cette fois le soutien de Ben Gourion, l'amène à négocier le plan Peel de 1937, première offre officielle de partager la Palestine en deux États indépendants, l'un juif et l'autre arabe : il est alors virtuellement le leader de l'État juif en gestation.

Pendant la Seconde Guerre mondiale, Weizmann met à nouveau sa compétence scientifique au service des Alliés, des deux côtés de l'Atlantique, comme il l'avait fait avec ses travaux sur l'acétone pendant la Première Guerre mondiale. Son fils Michel (1916-1942), pilote dans la RAF, est abattu dans le golfe de Gascogne. Le Livre blanc britannique anti-sioniste de 1939 venant en contradiction avec sa politique conciliatrice, Weizmann est pris à contrepied par les tendances sionistes «activistes» qui se font jour aussi bien au sein des organisations dissidentes, comme l'Irgoun Zvaï Leumi et le groupe Stern, que dans les éléments d'extrême-gauche du Palmach. Le violent affrontement entre sionistes et Britanniques est un échec personnel pour lui, et il n'est pas réélu à la présidence lors du Congrès sioniste de Bâle de décembre 1946. C'est là qu'il prononce sa formule célèbre : «On ne reçoit pas un État sur un plateau d'argent», qui sera transfigurée par un poème de Nathan Alterman. Là encore, Ben Gourion, après avoir contribué à minorer Weizmann, coopère avec lui pendant la délicate négociation à l'ONU sur la fin du mandat britannique, et il fait en sorte que le vieil homme soit élu président de l'État d'Israël à sa naissance, mais avec un statut seulement honorifique.

La diplomatie de Weizmann a fait preuve jusqu'au bout de son efficacité, ce fut le cas par exemple pour éviter, in extremis, l'abandon par les États-Unis du plan de partage adopté le 29 novembre 1947 par l'ONU et applicable dès le 15 mai 1948. Le secret de son influence se trouve dans ce coefficient personnel intellectuel et moral que l'on pourrait qualifier de charismatique - son ami Léon Blum l'a suggéré en écrivant: «J'ai jugé de l'empire extraordinaire qu'il exerçait sur les autres hommes par celui qu'il exerçait sur moi-même. Je n'ai jamais rien pu lui refuser $\gg{ }^{4}$.

\footnotetext{
${ }^{4}$ « Hommage à Weizmann », dans Léon Blum, L'œuvre de Léon Blum, 1947-1950, Paris, Albin Michel, 1963, p. 441.
} 


\section{Un acteur français méconnu de la Déclaration Balfour, Charles Dreyfus (1848-1935)}

La première rencontre entre Chaïm Weizmann et Arthur Balfour est bien antérieure aux négociations de 1917 : c'est au début de l'année 1906 que les deux hommes se sont rencontrés, à l'initiative d'un ami commun nommé Charles Dreyfus, dans des circonstances surprenantes. Charles Dreyfus est en effet à l'époque un des dirigeants de la Fédération sioniste du Royaume-Uni et, dans le débat en cours sur la question dite de l'Ouganda, il est partisan d'accepter la proposition du gouvernement britannique concernant un foyer juif en Afrique australe (dans le Kenya d'aujourd'hui), alors que Weizmann y est résolument opposé. Il pense qu'une conversation avec celui qui vient de quitter ses fonctions de Premier ministre pourrait faire changer d'avis son jeune ami. Et il suppose qu'Arthur Balfour y verra un moment de distraction dans la tension de sa campagne électorale dans la circonscription de Clayton (Nord Manchester) - qui se soldera finalement par une défaite. Or la conversation, prévue pour durer un quart d'heure, devait durer une heure et quart. Et le professeur de chimie arrivé deux ans plus tôt au RoyaumeUni, qui ne maîtrise pas encore, de son propre aveu, toutes les subtilités de la langue anglaise, défendit son point de vue avec une telle énergie que son interlocuteur fut fort intéressé d'entendre le point de vue d'un Juif russe, différent de celui des milieux de la grande bourgeoisie assimilationniste. Pour illustrer la force de l'attachement populaire juif à l'ancienne patrie perdue, Weizmann demanda à Balfour s'il accepterait qu'on lui propose de remplacer Londres par Paris. Devant l'étonnement de ce dernier qui lui dit: "Mais nous avons Londres!", Weizmann : répondit : « Mais nous avions Jérusalem à une époque où Londres n'était qu'un marécage ». " Vous ne rencontrez pas la bonne sorte de Juifs », expliqua-t-il à Balfour, qui dut arbitrer à nouveau, en 1917, entre les deux mêmes courants opposés 5 .

Cet épisode justifie que l'on en sache un peu plus sur Charles Dreyfus. Né à Rixheim, dans la banlieue de Mulhouse, élève de l'École de chimie de cette ville puis docteur en chimie de l'Université de Strasbourg, c'est à travers cette discipline scientifique que son chemin a croisé celui du futur président d'Israël. Embauché très jeune chez Dollfus-Mieg et $C^{\text {ie }}$, la grande fabrique mulhousienne du fil DMC qui existe encore de nos jours, il aurait, selon la légende familiale, trouvé une

\footnotetext{
${ }^{5}$ Chaïm Weizmann, Naissance d'Israël (Trial and Error), Paris, Gallimard, 1957, sq.
} 
teinture de bleu répondant précisément aux désirs de l'impératrice Eugénie, mais cette découverte aurait suscité la jalousie de ses collègues et son désir de changer d'emploi. La suite logique était de partir à 21 ans pour Manchester, ville si importante pour l'industrie textile, où il créa en 1876 (à 28 ans) la compagnie Clayton Aniline. Cette société est devenue le plus puissante de la ville et fournissait les tissus pour la moitié des véhicules circulant dans le pays ${ }^{6}$.

Le rôle personnel de Charles Dreyfus était important dans la recherche de procédés innovants, notamment en ce qui concerne les teintures. C'est dans cette spécialité qu'il eut l'occasion d'embaucher Chaïm Weizmann, à la fin de 1904, pour un emploi à temps partiel compatible avec le poste à l'Université de Manchester qu'il venait d'obtenir : l'arrangement permettant d'utiliser le laboratoire de la Faculté pour des expériences utiles à la firme qui l'employait, déboucha sur le dépôt en commun, par la firme et par le savant, d'une douzaine de brevets. Il s'agissait de produire artificiellement des teintures jaunes pour remplacer, lors de la guerre russo-japonaise de 1904, la production à partir de matières naturelles importées du Japon ${ }^{7}$.

Dreyfus et Weizmann s'étaient rencontrés en marge du congrès sioniste de Bâle en 1903. Ils avaient été mis en contact par l'imprimeur et poète hébraïque Joseph Massel, militant sioniste de premier plan à Manchester, et Weizmann avait déjà fait la connaissance du frère cadet de Charles Dreyfus, Yvan, chez le grand rabbin Wertheimer de Genève ${ }^{8}$. Il avait commencé par solliciter Dreyfus au sujet de son épouse Vera Weizmann, qui fut embauchée comme médecin à l'hôpital juif de la ville. Car Charles Dreyfus était une personnalité importante de la ville, engagé pour cet hôpital, pour la synagogue libérale, pour le mouvement sioniste dont il devint le président local, et aussi au conseil municipal où il fut élu au titre des tories. C'est en tant que président du comité tory de Manchester Est qu'il noua des liens avec Arthur Balfour et fut le président de son comité de campagne en 1906. Le jeune Chaïm était invité au domicile des Dreyfus en attendant que son épouse quitte Genève pour le rejoindre. Et les époux Dreyfus lui rendirent visite

\footnotetext{
${ }^{6}$ Professor Tudor Rickards (Manchester Business School), Manchester Evening News, 15 février 2007.

7 Bob Weintraub, «Charles Dreyfus (1848-1935), Yellow Dyes and the Balfour Declaration », Bulletin of the Israel Chemical Society, $\mathrm{n}^{\circ} 25$, octobre 2010, pp. 55-56.

${ }^{8}$ « Nécrologie - Docteur Ivan Dreyfus », Information juive, 16 mars 1975.
} 
lorsqu'il fut blessé à la main dans un accident de laboratoire et dut rester alité pendant six semaines.

Personnalité chaleureuse, Charles Dreyfus a gardé un lien fort avec sa famille en France où il choisit de passer les dernières années de sa $v^{2}{ }^{9}$. Son frère Yvan, médecin, eut lui aussi un parcours exceptionnel en France comme promoteur des soins aux blessés des deux guerres mondiales dans un cadre mutualiste moderne et sophistiqué. Il fut un héros de la Résistance, dont un des fils, déporté comme résistant, est mort à Buchenwald-Dora. On trouve un autre neveu de Charles Dreyfus, René Lévy (1875-1912), d'abord au laboratoire de recherches de la Clayton Aniline, puis parmi les dirigeants d'Air liquide, et finalement parmi les victimes du naufrage du Titanic ${ }^{10}$. Les Dreyfus de Mulhouse évoqués ici sont des cousins éloignés du capitaine Alfred Dreyfus.

\footnotetext{
9 «Charles Dreyfus» et Terry Zakine-Cerf, «Souvenirs de famille sur Charles Dreyfus », site du Center for Jewish Studies, University of Manchester.

${ }^{10}$ Site de la Médiathèque de la mer (Cherbourg), mediathèquedelamer.com/wp.../lacité-de-la-mer-fiches-passagers-titanic-rene-levy.pdf.
} 